\title{
Prevention of chemotherapy-induced nausea and vomiting: focus on fosaprepitant
}

\author{
Ian $\mathrm{N}$ Olver \\ The Cancer Council Australia, Sydney, \\ New South Wales, Australia
}

\begin{abstract}
Fosaprepitant is a prodrug of aprepitant, a neurokinin ${ }_{1}\left(\mathrm{NK}_{1}\right)$ receptor antagonist used in prophylactic antiemetic regimens used prior to cytotoxic chemotherapy. Fosaprepitant is being developed to provide a parenterally administered alternative to the orally administered aprepitant. Fosaprepitant is rapidly converted to aprepitant and an intravenous dose of $115 \mathrm{mg}$ is bioequivalent to $125 \mathrm{mg}$ orally, with similar plasma concentrations at 24 hours. In phase I and II trials fosaprepitant shows efficacy, but the large randomized efficacy studies have utilized aprepitant. When it is added to dexamethasone and a $5 \mathrm{HT}_{3}$ receptor antagonist on day 1 prior to chemotherapy aprepitant improves the control of acute post chemotherapy emesis and when continued on days 2 and 3 with dexamethasone it demonstrated even greater improvement in the control of delayed emesis. This has been shown with both cisplatin-containing regimens and those based upon cyclophosphamide and an anthracycline. Fosaprepitant is well tolerated with mild to moderate venous irritation being the only additional toxicity to those seen with oral aprepitant, and that is a function of dose, concentration, and infusion rate. Headaches are the other toxicity most commonly reported. Fosaprepitant can be used as a parenteral alternative to aprepitant in regimens to control chemotherapy-induced emesis.
\end{abstract}

Keywords: fosaprepitant, aprepitant, neurokinin ${ }_{1}$ receptor, emesis, chemotherapy

\section{Introduction}

The introduction of cytotoxic chemotherapy, and particularly cisplatin, was associated with nausea and vomiting that did not respond to conventional doses of the then available antiemetics, exemplified by metoclopramide and prochlorperazine. Of the factors that predicted nausea and vomiting the most significant was the cytotoxic drug, as each drug displayed a different emetic potential, and there were different mechanisms by which the cytotoxics could cause vomiting (Andrews et al 1998; Hesketh 1999). The most common pattern of nausea and vomiting is acute emesis which commences within hours of receiving chemotherapy and lasts over the first 24 hours. This can be followed by delayed emesis commencing near the beginning of the first day and often lasting for at least 5 days (Kris et al 1985). Those patients who experience severe postchemotherapy emesis are then prone to developing anticipatory emesis as a conditioned response (Morrow 1982). Patients who have poor control of post chemotherapy emesis also demonstrate deterioration in their quality of life (Osoba et al 1997).

It was the discovery that two important mechanisms for post-chemotherapy emesis were mediated through 5 hydroxytryptamine ${ }_{3}\left(5 \mathrm{HT}_{3}\right)$ and neurokinin ${ }_{1}\left(\mathrm{NK}_{1}\right)$ receptors and the development of antagonists that saw a great impact made upon the control of chemotherapy-induced vomiting and to a lesser extent nausea. The $5 \mathrm{HT}_{3}$ receptors, predominantly in the small bowel, were major mediators of acute emesis, and the first of the antagonists, ondansetron, when given prior to chemotherapy, revolutionized the control of post chemotherapy acute emesis. Ondansetron and dexamethasone controlled acute emesis in over 80\% patients (Gralla et al 1999). Patients, however, were still 
listing nausea and vomiting in their top three side effects even after the great improvement in the control of acute emesis (Boer-Dennert et al 1997). This was due to the incidence of delayed emesis which can occur in $20 \%-25 \%$ patients in the absence of acute emesis, and which was being underestimated by clinicians by up to $30 \%$ (Grunberg et al 2004).

Unfortunately, in only $50 \%$ patients was the delayed phase of emesis, caused by drugs such as cisplatin, controlled by ondansetron and dexamethasone. It was the dexamethasone that was the most active drug, suggesting that a different mechanism was responsible for delayed compared with acute emesis (Olver et al 1996).

Substance $\mathrm{P}$, a tachykinin, binds to $\mathrm{NK}_{1}$ receptors in the brain stem which send messages to the vomiting center and induce vomiting. Blocking the $\mathrm{NK}_{1}$ receptor lessens vomiting after cisplatin, and a variety of other emetic stimuli (Diemunsch and Grelot 2000).

With the development of the orally active $\mathrm{NK}_{1}$ receptor antagonist, aprepitant, it was found that when it was added to ondansetron and dexamethasone it improved the control of cisplatin-induced acute emesis, but when continued for 2 further days had a major impact on the control of the delayed phase of the post-chemotherapy emesis (Hesketh et al 2003; Poli-Bigelli et al 2003).

Fosaprepitant (L-758,298 or MK-0517) is a prodrug of aprepitant that can be administered intravenously and is converted into aprepitant within 30 minutes (Navari 2007).

\section{The pharmacology of fosaprepitant}

Fosaprepitant dimeglumine is a white powder which is freely water soluble and is a phosphoryl prodrug of aprepitant (Hale et al 2000). Its antiemetic properties are attributable to aprepitant, which is a selective neurokinin 1 (NK1) receptor antagonist with low affinity for NK2 and NK3 receptors (Watson et al 1998). It inhibits chemotherapy emesis by penetrating the brain and occupying central NK1 receptors for a sufficient duration to inhibit both the acute and delayed phases of emesis (Tattersall et al 2000). Antiemetic efficacy with aprepitant increases with receptor occupancy up until a dose of $125 \mathrm{mg}$ orally, but there is no greater benefit with higher doses (Hargreaves 2002).

Fosaprepitant $115 \mathrm{mg}$ given intravenously is bioequivalent to aprepitant $125 \mathrm{mg}$ given by mouth with similar plasma concentrations at 24 hours (Merck and Co Inc 2007). It has been trialed in single daily doses for up to 4 days. Fosaprepitant is converted to aprepitant within 30 minutes after the end of an infusion. Aprepitant is $95 \%$ bound to plasma proteins. In vitro studies show that aprepitant is metabolized in the liver primarily by CYP3A4, with minor metabolism by CYP1A2 and CYP2C19.

Preclinical toxicology studies of bolus fosaprepitant administered in seconds to dogs and rats showed that concentrations of $\leq 1 \mathrm{mg} / \mathrm{mL}$ were well tolerated. Concentrations up to $25 \mathrm{mg} / \mathrm{ml}$ at low doses (2-4 mg/ kg/day) were well tolerated in dogs but intermediate concentrations $(10 \mathrm{mg} / \mathrm{mL})$ given at higher doses $(32 \mathrm{mg} / \mathrm{kg} /$ day $)$ caused venous irritation (Lasseter et al 2007).

There have been seven metabolites identified in human plasma, which are only mildly active. Following a single intravenous dose of 14C-labeled fosaprepitant $57 \%$ of the radioactivity was recovered in the urine and $45 \%$ in the feces. The terminal half-life of aprepitant following the administration of aprepitant is around 14 hours (Merck and Co Inc 2007).

No dose adjustment is considered necessary based on gender, race, or age although there are no data available for fosaprepitant in patients less that 18 years old. Based on pharmacokinetics no dose adjustment is required for severe renal insufficiency including those patients on dialysis (Bergman et al 2005). Similarly there is no clinically meaningful difference in the pharmacokinetic parameters patients with mild to moderated hepatic insufficiency (Child-Pugh score 7-9) and no data on severe hepatic insufficiency.

\section{Drug interactions}

As a moderate inhibitor of CYP3A4, fosaprepitant/aprepitant should not be co-administered with drugs such as pimozide, terfenadine, astemizole, or cisapride where the inhibition may result in elevated concentrations of these drugs, with serious consequences. Similar care should be taken with drugs with a narrow therapeutic index that are metabolized by CYP3A4 such as cyclosporine, sirolimus, and tacrolimus. As fosaprepitant/aprepitant induces the metabolism of drugs metabolized by CYP2C9, the co-administration with drugs metabolized by this mechanism such as warfarin should be monitored carefully (Depre et al 2005). This is also the case for tolbutamide and phenytoin, which may result in them achieving lower plasma concentrations than desirable. The concentrations of oral contraceptives may be decreased with co-administration of aprepitant, so alternate contraception should be used.

Of the drugs to be given with fosaprepitant, there is no evidence of clinically meaningful interactions with $5 \mathrm{HT}_{3}$ antagonists, including palonosetron (Blum et al 2003; Shah et al 2005). There are a number of chemotherapy agents metabolized by CYP3A4 such as taxanes, etoposide, 
irinotecan, ifosfamide, imatininb, and vinca alkaloids. Oral aprepitant did not influence the pharmacokinetics of docetaxel or vinorelbine and there has been no obvious interaction clinically when administered with etoposide, vinorelbine and paclitaxel (Nygren et al 2005; Loos et al 2007). Aprepitant does inhibit cyclophosphamide bioactivation and thiotepa metabolism, but the effects are small (de Jonge et al 2005).

Aprepitant increases the AUC of dexamethasone, a substrate of CYP3A4 2.2 fold; therefore the co-administered doses of dexamethasone should be halved, as was done in the two phase III trials that established the efficacy of aprepitant in preventing high-dose cisplatin-induced emesis (McCrea et al 2003).

Drugs such as rifampicin and St John's Wort, strong CYP3A4 inducers, reduce the concentration of aprepitant/ fosaprepitant whereas a strong CYP3A4 inhibitor like ketoconazole can increase the AUC of aprepitant five-fold. Food effects on the absorption of aprepitant are minimized because of its nanoparticle formulation (Olver et al 2007).

\section{Early phase clinical trials of fosaprepitant}

In the initial development of aprepitant, two of the phase II trials used the intravenous prodrug L-758298, now called fosaprepitant. These trials were conducted in patients who were receiving their first ever dose of at least $50 \mathrm{mg} / \mathrm{m}^{2}$ cisplatin, which without antiemetic prophylaxis will be associated with acute nausea and vomiting in $100 \%$ of patients and delayed vomiting in up to $70 \%$ of patients (Kris et al 1996). In the first of these fosaprepitant at a dose of either $60 \mathrm{mg}$ or $100 \mathrm{mg}$ was compared with ondansetron $32 \mathrm{mg}$ given 1 hour before the chemotherapy. There was no significant difference in acute postchemotherapy emesis, with $37 \%$ on fosaprepitant and $52 \%$ on ondansetron having no emesis. In the delayed phase, $72 \%$ receiving fosaprepitant had no emesis compared with 30\% receiving ondansetron (Cocquyt et al 2001).

In the next study, fosaprepitant was studied in combinations. One group of patients received fosaprepitant $100 \mathrm{mg}$ with intravenous dexamethasone $20 \mathrm{mg}$ on day 1 and then oral aprepitant (MK-869) $300 \mathrm{mg}$ on days 2 to 5 . The second group had the same drugs on day 1 and placebo on days 2 to 5 and the third group received intravenous ondansetron $32 \mathrm{mg}$ added to dexamethasone on day 1 and placebo on days 2 to 5 . Acute emesis was best controlled by the ondansetron and dexamethasone group (83\%) compared with only $40 \%$ when the fosaprepitant groups were combined. However, the fosaprepitant groups did better in the delayed phase of the emesis with complete responses recorded for group 1 in
$59 \%$, for group 2 in 46\%, and for group 3 in 38\% (p $<0.05$ group 1 vs group 3) (van Belle et al 2002). These trials suggested that $\mathrm{NK}_{1}$ receptor antagonists were going to be best in combination antiemetic regimens.

Later, fosaprepitant was developed as an alternativee intravenous formulation to oral aprepitant. A study was performed to find the bioequivalent dose of fosaprepitant, in a polysorbate 80 vehicle, in terms of aprepitant AUC (area under the concentration time curve) to $125 \mathrm{mg}$ aprepitant (Lasseter et al 2007). The study was in 3 parts. In parts 1 and 2 fosaprepitant doses from 90 to $150 \mathrm{mg}$ were investigated, and based on those results 2 doses of fosaprepitant, 100 and $115 \mathrm{mg}$, were selected for the randomized open label crossover test of bioequivalence in part 3. Blood samples were collected over 72 hours following the drug administration for aprepitant assays. Patients ranged in age from 18 to 45 years. Across all three parts of the study 106 subjects received fosaprepitant doses ranging from 90 to 150 . There were no subjects discontinued because of adverse events. In parts 1 and 2 neither the $150 \mathrm{mg}$ dose nor the $90 \mathrm{mg}$ dose met the AUC bioequivalence criteria relative to $125 \mathrm{mg}$ aprepitant. The $115 \mathrm{mg}$ dose proved bioequivalent to aprepitant. It is rapidly converted to aprepitant with a half-life in the plasma of 2.3 minutes and complete conversion occurs within 30 minutes. It is not extensively distributed to the tissues. Although fosaprepitant resulted in a higher maximum concentration than aprepitant, the trough concentrations at 24 hours were equivalent, suggesting similar $\mathrm{NK}_{1}$ receptor occupancy. This suggests that fosaprepitant $115 \mathrm{mg}$ can be used interchangeably with $125 \mathrm{mg}$ aprepitant.

\section{Efficacy}

The efficacy data come from the trials of aprepitant. Initially there were 3 other phase II studies with aprepitant. A 351-patient placebo-controlled study in cisplatin-induced emesis studied 4 groups: the first used granisetron and dexamethasone on day 1 with placebo days 2 to 5 ; the second added in aprepitant on day 1 and then 2 to 5 ; the third arm used aprepitant the day before chemotherapy and then with dexamethasone on day 1 but without the granisetron, and then continued aprepitant days 1 to 5; and the final arm was similar but with the aprepitant and dexamethasone starting on day 1 (Campos et al 2001). This trial confirmed the need for a $5 \mathrm{HT}_{3}$ antagonist as part of triple therapy on day 1 for controlling the acute phase of emesis and confirmed the benefit of aprepitant in delayed emesis.

In a further phase II study of 159 patients, granisetron and dexamethasone was the standard treatment with other groups 
adding aprepitant to this on day 1 , then either continuing aprepitant until day 5 or using placebo form days 2 to 5 (Navari et al 1999). There was a significantly superior result for acute emesis in the two groups who received triple therapy on day $1(93 \%$ and $94 \%$ for complete control of emesis vs $67 \% \mathrm{p}<0.001)$ and in the delayed phase those groups receiving aprepitant were significantly better ( $82 \%$ and $78 \%$ vs $33 \%, \mathrm{p}<0.001)$ although the difference between just day 1 aprepitant and daily dosing for 5 days did not reach statistical significance.

A further phase II was designed to refine the dosing of aprepitant, adding to ondansetron plus dexamethasone $125 \mathrm{mg}$ aprepitant on day 1 and $80 \mathrm{mg}$ days 2 to 5 compared with $40 \mathrm{mg}$ on day 1 then $25 \mathrm{mg}$ on days 2 to 5 or placebo (Chawala et al 2003). Again the patients receiving aprepitant had superior control of acute and delayed emesis, but nausea was only better controlled in the delayed phase. The 125/80 mg dosing schedule yielded superior efficacy.

The efficacy of aprepitant was proven definitively in 2 phase II trials in patients receiving their first ever dose of cisplatin $\geq 70 \mathrm{mg} / \mathrm{m}^{2}$ over $\leq 3$ hours. The standard therapy was considered as ondansetron and dexamethasone on day 1 followed by dexamethasone on days 2 to 4 . The aprepitant arms added $125 \mathrm{mg}$ of oral aprepitant on day 1 and then $80 \mathrm{mg}$ days 2 and 3 giving just the dexamethasone on day 4 (Hesketh et al 2003; Poli-Bigelli et al 2003). Recording the Poli-Bigelli study first then the Hesketh study, the aprepitant arms were statistically significantly superior in overall complete response rates $(62.7 \%$ vs $43.3 \%, \mathrm{p}<0.001$ and $72.1 \%$ vs $52.3 \%, \mathrm{p}<0.001)$ and for acute emesis $(82.8 \%$ vs $68.4 \%, \mathrm{p}<0.001$ and $89.2 \%$ vs $78.1 \%$, p $<0.001)$. However, the most impressive response differences were in delayed emesis (67.7 \% vs 46.8\%, p < 0.001 and $74.4 \%$ vs $55.8 \%$, p $<0.001)$. Again, nausea was better controlled only in the delayed phase. The efficacy of aprepitant was maintained over 6 cycles.

A specific study of multiple cycles of high-dose cisplatin used ondansetron and dexamethasone for the acute phase and compared a placebo for the delayed phase with two different dosing schedules of aprepitant on days 1 to 5 (de Wit et al 2003). The complete response rate was maintained over 6 cycles in the aprepitant groups but not in the placebo group.

In randomized studies, the selection of the control arm is vital. Given that it had been common practice to continue the $5 \mathrm{HT}_{3}$ antagonist with the dexamethasone for 4 days, a study in 489 patients receiving high-dose cisplatin randomized them to either ondansetron and dexamethasone for on each day or triple therapy with aprepitant on day 1 followed by aprepitant and dexamethasone as given in the large randomized trials (Schmoll et al 2006). The aprepitant regimen yielded a significantly improved 5-day overall response rate $(72 \%$ vs $61 \%, \mathrm{p}=0.003)$ with a $9 \%$ improvement in protection from nausea and vomiting on day 1 and $11 \%$ on days 2 to 5 .

Following the studies with cisplatin-containing regimens, the efficacy of adding aprepitant to other chemotherapy regimens was trialed. In a large study 866 patients who were treated with cyclophosphamide and anthracylines, which as single agents have moderated emetic potential but as a combination arguably are of high emetic potential, were studied (Warr et al 2005). Patients were given oral ondansetron and dexamethasone with either aprepitant on days 1,2 , and 3 or just followed by 2 days of ondansetron. The overall 5-day complete response rate was $(50.8 \%$ vs $42.5 \%$, $\mathrm{p}=0.015)$ in favor of the aprepitant group. Nausea was not as well controlled as vomiting particularly in the delayed phase. The improved efficacy was maintained over 4 cycles of chemotherapy (Herrstedt et al 2005).

In a small trial, the role of aprepitant as a salvage therapy to add into the antiemetic regimen in subsequent cycles if patients had failed to respond to a $5 \mathrm{HT}_{3}$ receptor antagonist and dexamethasone was tested for both cisplatin and other chemotherapy (Oechsle et al 2006). The addition of aprepitant significantly improved activity.

There are no large studies of the efficacy of aprepitant in children or adolescents but case reports in adolescents are promising (Smith et al 2005).

As other new antiemetics are developed, combinations with aprepitant are tested. Palonosetron is a $5 \mathrm{HT}_{3}$ receptor antagonist with a longer duration of activity which has been reported as having better activity against the delayed emesis associated with chemotherapy of moderate emetic potential. After determining that there were no pharmacokinetic problems with the combination, aprepitant with palonosetron and dexamethasone was found to be highly effective in preventing chemotherapy-induced nausea and vomiting in the days following the administration of cyclophosphamide chemotherapy of moderate emetic potential (Shah et al 2005; Grote et al 2006).

\section{Safety and tolerability}

Fosaprepitant and aprepitant are very well tolerated. In the initial phase 1 studies with aprepitant or its prodrug the adverse events that were reported more commonly for aprepitant groups over placebo were fatigue, somnolence, dizziness, 
flushing, nausea, hiccups, headache, and menstrual problems. In the trials of L-758298 compared with ondansetron the only significant difference in toxicities was more diarrhoea with the prodrug than ondansetron, which itself would tend toward producing constipation (Cocquyt et al 2001; van Belle et al 2002). Most studies administered aprepitant as part of an antiemetic regimen which often included $5 \mathrm{HT}_{3}$ receptor antagonists, so it has been difficult to isolate the side effects specifically due to aprepitant. In the two large phase III trials the incidence of drug-related side adverse events for the aprepitant arm was $19.5 \%$ vs $14.4 \%$ in the control arm in the Poli-Bigelli study and $14.6 \%$ vs $11.0 \%$ in the Hesketh study (Hesketh et al 2003; Poli-Bigelli et al 2003). The most reported side effects were asthenia, anorexia, diarrhea, headaches, and hiccups. In the Warr study there was more constipation in the control arm and more dyspepsia in the aprepitant arm (Warr et al 2005).

In the study to establish the bioequivalence of fosaprepitant there were no serious adverse events or laboratory toxicities (Lasseter et al 2007). Headache and infusion site symptoms were the most reported events, but these were only of mild to moderate intensity. Fosaprepitant is tolerable at $1 \mathrm{mg} / \mathrm{mL}$ infused over 15 to 30 minutes but was found to cause irritation at $25 \mathrm{mg} / \mathrm{mL}$ at doses of $50 \mathrm{mg}$ or $100 \mathrm{mg}$ infused over 30 seconds.

\section{Patient quality of life and satisfaction}

The impact on patients of chemotherapy-induced nausea and vomiting goes beyond those side effects to be associated with a measurable deterioration in their global quality of life (Osoba et al 1997). More specifically the patients experiencing emesis also have more fatigue, anorexia, and insomnia.

In the large phase III trials of aprepitant in combination regimens for cisplatin and non-cisplatin induced emesis, a quality of life scale, the Functional Living Index Emesis (FLIE), was used (Martin et al 2003). The arms of the studies that contained aprepitant reported minimal or no impact of the emesis on daily life compared with those just receiving a $5 \mathrm{HT}_{3}$ antagonist and dexamethasone $(74.4 \%$ vs $63.5 \%$ in the Poli-Bigelli study and $70.4 \%$ vs $55.6 \%$ in the Hesketh study) (Hesketh et al 2003; Poli-Bigelli et al 2003). In subgroup analyses this improvement in maintaining daily life activities was independent of sex and independent of age (Ma et al 2003; Martin et al 2003; Hesketh et al 2006). A similar outcome was reported in the Warr study where cyclophosphamide and an anthracycline was the chemotherapy, and those reporting minimal or no impact of emesis and quality of life were $63.5 \%$ on aprepitant vs $55.6 \%$ without, $\mathrm{p}=0.019$ ).
Cost effectiveness is also an important consideration for patients. It has been calculated that the routine use of aprepitant in antiemetic prophylaxis is cost effective in those situations where the likelihood of delayed emesis is high or the need will exist later for costly rescue medications (Moore et al 2007).

\section{Conclusions}

Fosaprepitant, a prodrug of aprepitant, is being developed as an intravenous alternative to oral aprepitant. It is rapidly converted to aprepitant after administration. The efficacy is the same as that of aprepitant. It is very well tolerated, with venous irritation being specific to this formulation and headache being the most frequent of the other toxicities. It seems that it is able to be used interchangeably with aprepitant and has been safely administered in single- and multiple-day schedules.

For example, in triple antiemetic therapy, fosaprepitant could be administered on day 1 with other antiemetics before intravenous chemotherapy and then on days 2 and 3 the $\mathrm{NK}_{1}$ therapy could be continued with oral aprepitant. An intravenous formulation may be more convenient in some circumstances and in some jurisdictions there are differing reimbursements for intravenous and oral drugs which may make an intravenous formulation more desirable, particularly if the income was being used to fund additional patient services.

Medical indications would include patients with severe mucositis or difficulty swallowing or any gastrointestinal disturbance that would make oral therapy problematic.

Fosaprepitant provides an intravenous alternative to oral formulations of $\mathrm{NK}_{1}$ receptor antagonists that is safe and effective.

\section{References}

Andrews PI, Naylor RJ, Joss RA. 1998. Neuropharmacology of vomiting and its relevance to anti-emetic therapy. Consensus and controversies. Support Care Cancer, 6:197-203.

Bergman AJ, Marbury T, Fosbinder T, et al. 2005. Effect of impaired renal function and haemodialysis on the pharmacokinetics of aprepitant. Clin Pharmacokinet, 44:637-47.

Blum RA, Majumdar A, McCrea J, et al. 2003. Effects of aprepitant on the pharmacokinetics of ondansetron and granisetron in healthy subjects. Clin Ther, 25:1407-19.

Boer-Dennert M, Wit R, Schmitz PJ, et al. 1997. Patient perceptions of the side effects of chemotherapy: the influence of the 5-HT3 antagonists. Br J Cancer, 76:1055-61.

Campos D, Pereira JR, Reinhardt RR, et al. 2001. Prevention of cisplatininduced emesis by the oral neurokinin-1 antagonist, MK-869, in combination with granisetron and dexamethasone or with dexamethasone alone. J Clin Oncol, 19:1759-67.

Chawala SP, Grunberg SM, Gralla RJ, et al. 2003. Establishing the dose of the oral $\mathrm{NK}_{1}$ antagonist aprepitant for the prevention of chemotherapyinduced nausea and vomiting. Cancer, 97:2290-300. 
Cocquyt V, Van Belle S, Reinhardt RR, et al. 2001. Comparison of L-758,298 a prodrug for the selective neurokinin-1 antagonist, L-754,030, with ondansetron for the prevention of cisplatin induced emesis. Eur J Cancer, 37:835-42.

De Jonge ME, Huitema AD, Holtkamp MJ, et al. 2005. Aprepitant inhibits cyclophosphamide bioactivation and thiotepa metabolism. Cancer Chemother Pharmacol, 56:370-8.

Depre M, Van Hecken A, Oeyen M, et al. 2005. Effect of aprepitant on the pharmacokinetics and pharmacodynamics of warfarin. Eur J Clin Pharmacol, 61:341-6.

De Wit R, Herrstedt J, Rapoport B, et al. 2003. Addition of the oral $\mathrm{NK}_{1}$ antagonist aprepitant to standard antiemetics provides protection against nausea and vomiting during multiple cycles of cisplatin-based chemotherapy. J Clin Oncol, 21:4105-19.

Gralla RJ, Osoba D, Kris MG, et al. 1999. Recommendations for the use of antiemetics: evidence-based, clinical practice guidelines. J Clin Oncol,17: 2971-94.

Grote T, Hajdenberg J, Cartmell A, et al. 2006. Combination therapy for chemotherapy-induced nausea and vomiting in patients receiving moderately emetogenic chemotherapy: palonosetron, dexamethasone, and aprepitant. J Support Oncol, 4:403-8.

Grunberg SM, Deuson RR, Mavros P, et al. 2004. Incidence of chemotherapy-induced nausea and emesis after modern antiemetics. Cancer, 100:2261-8

Hale JJ, Mills SG, MacCoss M, et al. 2000. Phosphorated morpholine acetal human neurokinin-1 receptor antagonists as water soluble prodrugs. $J$ Med Chem, 43:1234-41.

Hargreaves R. 2002. Imaging substance P receptors (NK1) in the living human brain using positrom emission tomography. J Clin Psychiatry, 63:18-24.

Herrstedt J, Muss HB, Warr DG, et al. 2005. Efficacy and tolerability of aprepitant for the prevention of chemotherapy-induced nausea and emesis over multiple cycles of moderately emetogenic chemotherapy. Cancer, 104:1548-55.

Hesketh PA. 1999. Defining the emetogenicity of cancer chemotherapy regimens: relevance to clinical practice. Oncologist, 4:191-6.

Hesketh PJ, Grunberg SM, Gralla RJ, et al. 2003. The oral neurokinin-1 antagonists aprepitant for the prevention of chemotherapy-induced nausea and vomiting: a multinational, randomized, double-blind, placebo-controlled trial in patients receiving high-dose cisplatin. The aprepitant protocol 052 study group. J Clin Oncol, 21:4112-9.

Hesketh PJ, Grunberg SM, Herrstedt J, et al. 2006. Combined data from two phase III trials of the NK1 antagonist aprepitant plus a 5HT 3 antagonist and a corticosteroid for prevention of chemotherapy-induced nausea and vomiting: effect of gender on treatment response. Support Care Cancer, 14:354-60.

Kris MG, Gralla RJ, Clark RA, et al. 1985. Incidence, course and severity of delayed nausea and vomiting following the administration of highdose cisplatin. J Clin Oncol, 3:1379-84.

Kris MG, Cubeddu LX, Gralla RJ, et al. 1996. Are more antiemetic trials with a placebo necessary? Report of patient data from randomized trials of placebo antiemetics with cisplatin. Cancer, 78:2193-8.

Lasseter KC, Gambale J, Jin B, et al. 2007. Tolerability of fosaprepitant and bioequivalency to aprepitant in healthy subjects. J Clin Pharmacol, 47:834-40.

Loos WJ, de Wit R, Freedman SJ, et al. 2007. Aprepitant when added to a standard antiemetic regimen consisting of ondansetron and dexamethasone does not affect vinorelbine pharmacokinetics in cancer patients. Cancer Chemother Pharmacol, 59:407-12.

McCrea JB, Majumdar AK, Goldberg MR, et al. 2003. Effects of the neurokinin 1 receptor antagonist aprepitant on the pharmacokinetics of dexamethasone and methylprednisolone. Clin Pharm Ther, 74:17-24.

Ma JG, Martin AR, Carides AD, et al. 2003. The oral NK1 antagonist, aprepitant, is effective in reducing impact of chemotherapy-induced nausea and vomiting on daily life in both younger and older patients receiving highly emetogenic chemotherapy. Proc Am Soc Clin Oncol, 22:785.
Martin AR, Carides AD, Pearson JD, et al. 2003. Functional relevance of antiemetic control. Experience using the FLIE questionnaire in a randomised study of the NK-1 antagonist aprepitant. Eur J Cancer, 39:1395-401.

Merck and Co Inc. 2007. Product Information. Emend IV ${ }^{\mathrm{TM}}$ (fosaprepitant dimeglumine, MSD) Approved by TGA Therapeutics Goods Administration 14 Aug 2007.

Moore S, Tumeh J, Wojtanowski S, et al. 2007. Cost-effectiveness of aprepitant for the prevention of chemotherapy-induced nausea and vomiting associated with highly emetogenic chemotherapy. Value Health, 10:23-31.

Morrow GR. 1982. Prevalence and correlates of anticipatory nausea and vomiting in chemotherapy patients. J Natl Cancer Instit, 68:585-93.

Navari RM, Reinhardt RR, Gralla RJ, et al. 1999. Reduction of cisplatininduced emesis by a selective neurokinin-1 receptor antagonist. $N$ Engl JMed, 340:190-5.

Navari RM. 2007. Fosaprepitant (MK-0517): a neurokinin-1 receptor antagonist for the prevention of chemotherapy-induced nausea and vomiting. Expert Opin Investig Drugs, 16:1977-85.

Nygren P, Hande K, Petty KJ, et al. 2005. Lack of effect of aprepitant on the pharmacokinetics of docetaxel in cancer patients. Cancer Chemother Pharmacol, 55:609-16.

Oechsle K, Muller MR, Hartmann JT, et al. 2006. Aprepitant as salvage therapy in patients with chemotherapy-induced nausea and emesis refractory to prophylaxis with 5-HT (3) antagonists and dexamethasone. Onkologie, 29:557-61.

Olver I, Paska W, Depierre A, et al. 1996. A multicentre, double-blind study comparing placebo, ondansetron and ondansetron plus dexamethasone for the control of cisplatin-induced delayed emesis. Ann Oncol, 7:945-52.

Olver I, Shelukar S, Thompson K. 2007. Nanomedicines in the treatment of emesis during chemotherapy: focus on aprepitant. Int J Nanomedicine, 2:13-18.

Osoba D, Zee B, Warr D, et al. 1997. Effect of postchemotherapy nausea and vomiting on health-related quality of life. The Quality of life and symptom control committees of the National Cancer Institute of Canada Clinical Trials Group. Support Care Cancer, 5:307-13.

Poli-Bigelli S, Rodrigues-Pereira J, Carides AD et al. 2003. Addition of the neurokinin 1 antagonist aprepitant to standard antiemetic therapy improves control of chemotherapy-induced nausea and vomiting: results from a randomized, double-blind, placebo-controlled trial in Latin America. Cancer, 97:3090-8.

Schmoll HJ, Aapro MS, Poli-Bigelli, et al. 2006. Comparison of aprepitant regimes with a multiple day ondansetron regimen, both with dexamethasone, for antiemetic efficacy in high-dose cisplatin treatment. Ann Oncol, 17:1000-6.

Shah AK, Hunt TL, Gallagher SC, et al. 2005. Pharmacokinetics of palonosetron in combinations with aprepitant in healthy volunteers. Curr Med Res Opin, 21:595-601.

Smith AR, Repka TL, Weigel BJ. 2005. Aprepitant for the control of chemotherapy induced nausea and vomiting in adolescents. Pediatr Blood Cancer, 45:857-60.

Tattersall FD, Rycroft W, Cumberbatch M, et al. 2000. The novel NK1 receptor antagonist MK-0869 (L-754,030) and its water soluble phosphoryl prodrug, L-758,298, inhibit acute and delayed cisplatin-induced emesis in ferrets. Neuropharmacology, 39:652-63.

Van Belle S, Lichinister MR, Navari RM, et al. 2002. Prevention of cisplatin-induced acute and delayed emesis by the selective neurokinin-1 antagonists, L-758,298 and MK-869: a randomized controlled trial. Cancer, 94:3032-41.

Warr DG, Hesketh PJ, Gralla RJ, et al. 2005. Efficacy and tolerability of aprepitant for the prevention of chemotherapy-induced nausea and vomiting in patients with breast cancer after moderately emtogenic chemotherapy. J Clin Oncol, 23:2882-30.

Watson JW, Gonsalves SF, Rossa AA, et al. 1998. The antiemetic effects of CP-99,994 in the ferret and dog: role of the NK-1 receptor. $B r J$ Pharmacol, 115:84-94. 